\title{
COVID-19 INTERSTITIAL PNEUMONIA (CASE REPORT)
}

\author{
Ashcheulova T., Volik M. \\ Kharkiv National Medical University, Ukraine \\ https://doi.org/10.35339/ic.7.4.174-179
}

\begin{abstract}
A clinical case of treatment of a 54-year-old man with several risk factors, including diabetes, obesity, hypertension, with moderate COVID-19 bilateral pneumonia is presented. His clinical symptoms and signs at presentation included combinations of fever, nonproductive cough, and shortness of breath. Initial chest radiograms faid to show any changes, but chest CT revealed ground glass opacities (GGOs) with fuzzy contours, parenchyma damage (27\%). He was treated according to the Guidelines for diagnosis and treatment of COVID-19 cases (the Order of Ministry of Health of Ukraine No. 762). After a 12-day treatment, the damaged area reduced to $5 \%$ with fibrosis.
\end{abstract}

Keywords: SARS-CoV-2, ground glass opacities, cytokine storm, bilateral pneumonia, fibrosis, interstitial pneumonitis.

\section{Introducton}

COronaVIrus Disease 2019 (COVID-19) is an acute respiratory pathology caused by Severe Acute Respiratory Syndrome coronavirus-2 (SARS-CoV-2) which has turned into a pandemic affecting many countries of the world and rapidly spreading throughout Ukraine.

By the early 21 st century, epidemic coronaviruses were not considered to be virtually dangerous. SARS was first reported in Asia in February 2003 and get around to more than two dozen countries in North America, South America, Europe, and Asia. According to the World Health Organization (WHO), about 8,098 people worldwide became sick and 774 died because of SARS-CoV-1 [1].

Despite the fact that SARS-CoV-1 is pretty similar to SARS-CoV-2, both of them are betacoronaviruses and are known to enter human cells through the angiotensin-converting-enzyme 2 receptor with human-to-human transmission by close contacts [2]. Older persons with related diseases such as hypertension, heart or lung disease, diabetes or cancer, are more likely to be affected [3]. Comparing with statistical data,

Corresponding Author:

Mariia Volik, MD, PhD student of the Department

of Propaedeutics of Internal Medicine № 1,

Basis of Bioethics and Biosafety, Kharkiv National

Medical University, Kharkiv, Ukraine.

E-mail: mariuena.amboto@gmail.com
SARS-CoV-2 is more contagious and cause greater morbidity. Thus, up to 27.10.2020, there were +6677 new cases per day, 355601 laboratory confirmed cases of the disease, 6590 fatalities, 145336 recovered in Ukraine [4].

The onset of COVID-19 has been found since late December 2019 in Wuhan City, China. The patients with fever, malaise, dry cough, and dyspnea were diagnosed with viral pneumonia that was called later Wuhan pneumonia because of the area symptoms. The causative agent is a novel the seventh member of the coronavirus family according to the genome sequencing. On 12 February 2020 the WHO officially named this infection COVID-19. Later, the International Committee on Taxonomy of Viruses on 12 January 2020 officially termed the virus SARS-CoV-2 based on phylogeny, taxonomy and established practice. Beginning in China, COVID-19 rapidly spread to other countries worldwide. On 11 March 2020, the WHO characterized COVID-19 as a pandemic, similar to 1918 Spanish flu (H1N1), 1957 Asian flu (H2N2), 1968 Hong Kong flu (H3N2), and 2009 Pandemic flu (H1N1), which caused an estimated 50 million, 1.5 million, 1 million, and 300,000 human deaths, respectively [5].

Despite the fact, that about $80 \%$ COVID-19 patients recover spontaneously without the need for hospitalization, in about one in five cases, it occurs in severe form with respiratory failure. Risk factors for complications of COVID-19 
include older age ( $>65$ years), cardiovascular disease, chronic lung disease, hypertension, diabetes, and obesity [2].

The new COVID-19 pneumonia has longer latent period (14-21 days vs. 7-8), during which the patient does not experience symptoms, but can spread virus. Such general symptoms as elevated body temperature up to $38^{\circ} \mathrm{C}$, dry cough and fatigue are not red flags for the majority of infected people leading to late diagnosis. Inappropriate treatment measures increase the frequency of more advanced forms of disease. Moreover, after full recovering, the person can have PCR positive test during 2-3 weeks.

Typical bacterial pneumonia is known to cause lung inflammation in which alveoli walls are damaged and alveoli are filled with exudate, thus causing pulmonary tissue consolidation. Such picture can be seen easily in the X-ray examination of the thoracic organs. At the same time, the coronavirus activated only septal interstitial tissue. The infiltrate accumulates in the interstitial deposition. Lung damage caused by COVID-19 is not just viral-bacterial pneumonia. It is more appropriate to speak about diffuse parenchyma disease as an expression of interstitial pneumonitis. Everything described compose the nonspecific picture of a "clear" X-ray film. Such type of inflammation can be seen only using a CT scan. That is why Computed Tomography (CT) is used as an important complement for the diagnosis of COVID-19 pneumonia [6].

Despite the fact that a number of guidelines have already been approved, there is no general rule for the treatment of the COVID-19 pneumonia, moreover the treatment methods are constantly being reviewed. Internists and family physicians are still asking questions about an effective treatment. Therefore, we presented a clinical case of successful treatment of a patient with moderate COVID-19 pneumonia that can help doctors of many specialties to understand the issues of diagnosis and treatment as well as control of the COVID-19 infection.

\section{Clinical case}

A 54-year-old man with bilateral pneumonia was admitted to pulmonology department of Kharkiv City Clinical Hospital No. 13 with bilateral chest pain that intensified with inspiration, nonproductive cough, fever of $39^{\circ} \mathrm{C}$, weakness, and malaise.

The patient felt acutely ill 9 days before the admission when the body temperature elevated up to $37.5^{\circ} \mathrm{C}$ and weakness occurred, followed by a nonproductive cough, bilateral chest pain aggravated by cough. About this time, he began to experience dyspnea, which progressively worsened; his body temperature elevated to $39.3^{\circ} \mathrm{C}$. Despite paracetamol treatment, the chest pain, the cough and fever persisted. Few days later, his condition was aggravated by shortness of breath. Thus, he was admitted to the pulmonology department for evaluation of his condition.

Medical history

The patient denied neurological, psychiatric, oncology diseases, myocardial infarction, stroke, hepatitis, HIV, infectious diseases in himself and other relatives. There was no history of previous lung diseases. He had a 15-year history of hypertension and 5-year history of type 2 diabetes mellitus. There were no injuries and surgeries. The patient did not smoke, used alcohol and drugs. There was no history of hereditary diseases. The patient denied allergic disease and response to blood transfusion, introduction of serums, medicines, a variety of nutrients, cosmetics.

Physical examination

On examination, his condition was moderately sever. His consciousness was clear, the patient was oriented. His posture was active with restriction, there was orthopnea. His habitus was characterized by incorrect body built with height of $175 \mathrm{~cm}$ and weight of $110 \mathrm{~kg}$, hypersthenic constitution. His skin was warm and moist, there was acrocyanosis, with intact turgor. There was lower extremity edema. The lymph nodes were impalpable. His musculoskeletal system was developed in accordance with the sex and age. The chest was hypersthenic, the supra- and subclavicular fossae were moderately pronounced, the direction of the ribs was moderately slanting, there was no deformation of the sternum. The chest was symmetrical, his respiratory rate was 27 per minute, rhythmic, of moderate depth, and the both parts of the chest equally took part in the breathing act. Comparative percussion of the lungs in supra- and subclavicular regions, over the anterior surface of the chest wall, in the axillary regions, in supra-, inter- and subscapular regions demonstrated dull sound. Auscultation of the lungs showed harsh breathing, bilateral crackles in the supraclavicular and suprascapular regions. The heart rhythm was regular, the cardiac murmurs were not heard. His heart rate was 117 beats per minute, pulse rate was 117 per minute, there was no pulse deficiency. His blood pressure was 110/ $90 \mathrm{~mm} \mathrm{Hg}$, body temperature $38.2^{\circ}$ degrees Celsius, with $93 \%$ oxygen saturation. His tongue was white and wet. The abdomen was spherical, 
enlarged in size, symmetrical. It was soft and painless to superficial tentative palpation. Pasternatsky's sign was negative on the both sides. The bladder and bowel habits were norm

Laboratoty Investigations

The blood test demonstrated leukopenia, increased ERS and monocytosis. Other laboratory findings are presented in table 1.

Table 1

\begin{tabular}{|l|c|c|}
\hline \multicolumn{1}{|c|}{ Parameter } & Result & Reference Ranges \\
\hline Hemoglobin & 118 & $120-160 \mathrm{~g} / \mathrm{l}$ \\
\hline RBC & $4.3^{*} 10^{12}$ & $3.9-5.0^{*} 10^{12} / \mathrm{l}$ \\
\hline WBC & $3.2^{*} 10^{9}$ & $4.0-9.0^{*} 10^{9} / \mathrm{I}$ \\
\hline ERS & 38 & $1-15 \mathrm{~mm} / \mathrm{h}$ \\
\hline Band & 4 & $1.0-6.0 \%$ \\
\hline Segmented & 56 & $47.0-72 \%$ \\
\hline Eosinophils & 1 & $0.5-5.0 \%$ \\
\hline Lymphocytes & 30 & $19.0-37.0 \%$ \\
\hline Monocytes & 9 & 3.0 \\
\hline
\end{tabular}

The blood levels of C-reactive protein, procalcitonin, ferritin, IL-6 were increased; other laboratory findings are shown in table 2.

Table 2

\section{Blood biochemistry}

\begin{tabular}{|l|c|c|}
\hline & Result & Reference Ranges \\
\hline CRP & $144 \mathrm{mg} / \mathrm{ml}$ & $>3.0 \mathrm{mg} / \mathrm{ml}$ \\
\hline Procalcitonin & $134 \mathrm{ng} / \mathrm{ml}$ & $>2 \mathrm{ng} / \mathrm{ml}$ \\
\hline Ferritin & $468 \mathrm{ng} / \mathrm{ml}$ & $346 \mathrm{ng} / \mathrm{ml}$ \\
\hline D-dimer & $115.28 \mathrm{ng} / \mathrm{ml}$ & $0-250 \mathrm{ng} / \mathrm{ml}$ \\
\hline IL-6 & $189 \mathrm{pg} / \mathrm{ml}$ & $7 \mathrm{pg} / \mathrm{ml}$ \\
\hline AST & $16 \mathrm{U} / \mathrm{I}$ & $<38 \mathrm{U} / \mathrm{l}$ \\
\hline ALT & $63 \mathrm{U} / \mathrm{I}$ & $<40 \mathrm{U} / \mathrm{I}$ \\
\hline Bilirubin total & $11.2 \mu \mathrm{mol} / \mathrm{l}$ & $5-21 \mu \mathrm{mol} / \mathrm{l}$ \\
\hline Bilirubin direct & $4.1 \mu \mathrm{mol} / \mathrm{l}$ & $2.2-5.13 \mu \mathrm{mol} / \mathrm{I}$ \\
\hline Bilirubin indirect & $7.1 \mu \mathrm{mol} / \mathrm{l}$ & $6.3-15.4 \mu \mathrm{mol} / \mathrm{I}$ \\
\hline BUN & $6.88 \mu \mathrm{mol} / /$ & $2,0-6,7 \mu \mathrm{mol} / \mathrm{l}$ \\
\hline Creatinine & $95.5 \mu \mathrm{mol} / \mathrm{I}$ & $53,0-97,2 \mu \mathrm{mol} / /$ \\
\hline Glycosylated hemoglobin & $9.06 \%$ & $5-7 \%$ \\
\hline Blood glucose & $7.1 \mathrm{mmol} / /$ & $3.3-5.5 \mathrm{mmol} / \mathrm{I}$ \\
\hline
\end{tabular}

It can be seen that the typical of laboratory findings in the hospitalized COVID-19 patient showed leukopenia and elevated levels of such inflammatory indices as C-reactive protein, IL-6, procalcitonin and ferritin, which are related to "cytokine storm". Cytokine storm is a systemic inflammatory response to infections and leads to excessive activation of immune cells and the generation of pro-inflammatory cytokines like IL-6, IL-1, TNF- $\alpha$, and interferon [7]. Cytokines are an essential part of the inflammatory process produced by innate macrophages, dendritic cells, natural killer cells and the adaptive T- and B-lymphocytes. However, atypical uncontrolled anti-viral immune response results in continuous activation of immune cells, lymphocytes, and macrophages, which produce immense amounts of cytokines, resulting in a cytokine storm with destructive effects on endothelial cell, vascular barrier, capillary damage, diffuse alveolar damage, multiorgan failure. The consequence of the cytokine storm is lung injury that can progress into ARDS [8].

SARS-CoV-2 infection was confirmed by means of polymerase-chain-reaction (PCR). The PCR test for the qualitative detection of nucleic acid from SARS-CoV-2 in upper and lower respiratory regions is most frequently used and reliable comparing with serological tests in identifying IgM and IgG. Either IgG or total antibodies (IgM + IgG) must be calculated because IgM to SARS-CoV-2 are weak indicators, which can lead to false negative results. Moreover, the serological test to SARS-CoV-2 is best performed at least 2 weeks from the onset of the first symptoms, better 3-4 weeks from the beginning. This is due to the fact that many people with COVID-19 have no antibodies in the first two weeks [9].

Clinical urinalysis demonstrated the following: color - yellow, transparency - moderate, urine ratio $1020, \mathrm{pH}-5.5$, glucose -1.7 , ketone bodies + , protein - no, leukocytes $-3-5$.

Instrumental investigations

The oxygen saturation using pulse oximeter was $93 \%$.

$\mathrm{X}$-ray with no syndromes on it is shown in fig. 1 .

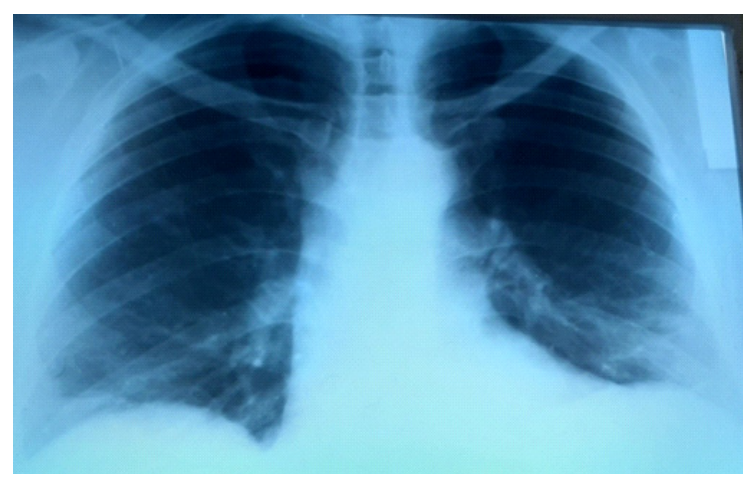

Fig. 1. X-ray film

Two days later, chest CT scan without intravenous contrast was performed which demonstrated low-intensity compaction centers looking like ground glass opacities (GGOs) with fuzzy contours, places of drainage. In the segments on the left and in the basal sections of both lungs, there were the signs of linear fibrosis. Lung tissue damage made $27 \%$ (fig. 2).

The key point ofCOVID-19 is bilateral presence of patchy ground glass opacities (GGOs) that may coalesce into dense, consolidated lesions, with a predominantly peripheral distribution under the pleura and along the bronchovascular bundles. In addition to GGOs and consolidations, COVID-19 


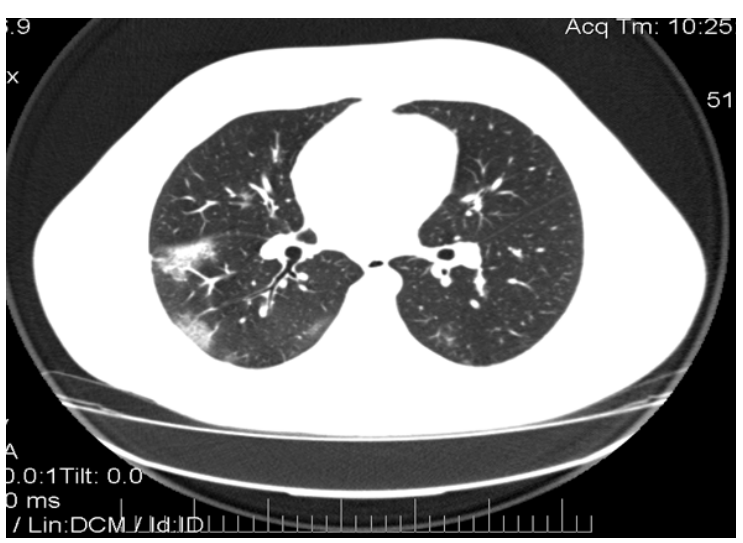

Fig. 2. Chest CT without contrast

pneumonia may show other CT findings or patterns such as interstitial thickening, crazypaving pattern, "reversed halo sign", "halo sign", and airway and vascular changes. These may help to differentiate COVID-19 pneumonia from other forms of pneumonia [10].

Electrocardiography (ECG) showed sinus tachycardia, 117 beats per minute, electrical axis deviation to the left (fig. 3).
The blood saturation was maintained at $97 \%$ using oxygen therapy. Comparison of the saturation with the respiratory rate showed that tachypnea correlated with the saturation. Adequate water balance up to 1.5 liters per day was maintained.

The treatment was performed according to the Guidelines for diagnosis and treatment of COVID-19 cases (the Order of Ministry of Health of Ukraine No. 762). The patient was administered empirical antibiotic treatment based on the level of procalcitonin, i.e. third-generation cephalosporin $1 \mathrm{~g}$ tid IV and azithromycin $500 \mathrm{mg}$ po once/day as well as a preventive doze of lowmolecular heparin (anticoagulants) 0.4 tid because of increased risk of a blood clot development, the doze was calculated with accordance to D-Dimer [12]. Systemic corticosteroids included dexamethasone $8 \mathrm{mg}$ IV per day due to respiratory failure. Additionally, he received antioxidant therapy such as L-arginine aspartate $100 \mathrm{ml} \mathrm{IV,}$ ascorbic acid $4 \mathrm{ml} \mathrm{IV}$ and rheosorbilact solution $200 \mathrm{ml} \mathrm{IV}$ to reduce intoxication. Treatment of comorbid pathology included combination of

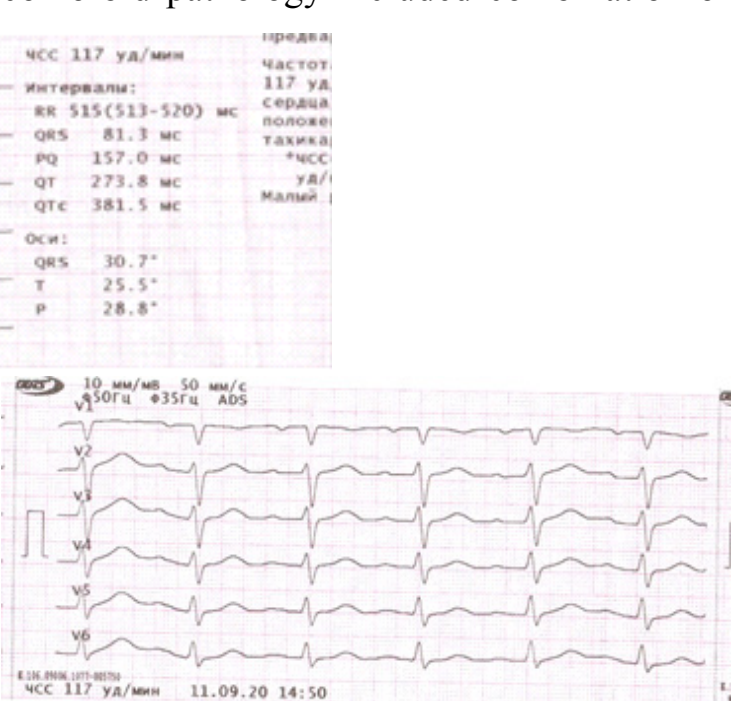

Fig. 3. ECG

Final diagnosis

Bilateral polysegmental interstitial moderate COVID-19 pneumonia with stage 2 respiratory failure was made. SARS-Co-2 RNA were identified on 11.09.2020.

The patient was diagnosed with coronary heart disease, cardiosclerosis, grade 2 hypertension of high risk, II A heart failure, stage 2 type 2 diabetes mellitus.

Treatment

The patient required oxygen supply on the first day of hospitalization because of hypoxemia. Supplemental oxygen was administered through a nasal cannula at a rate of 5 liters per minute.
Perindopril/Indapamide/Amlodipine $8 / 2.5 / 10$ po once/day (hypertension), Metformin $1,000 \mathrm{mg}$ tid (type 2 diabetes mellitus). The patient was placed in the prone position in bed.

Outcome

The following 2 days, his fever elevated to $39.2^{\circ} \mathrm{C}$. Chills, cough persisted; dyspnea increased; headache, diarrhea developed. The rate of supplemental oxygen was increased to 10 liters per minute. On the third day the initial antibiotic treatment was changed to fluoroquinolones (levofloxacin) $500 \mathrm{mg}$ IV without any other changes. Normal blood glucose level at about 6.1 $\mathrm{mmol} / \mathrm{l}$ was maintained using Metformin, because 
typically glucose level in COVID-19 patients with type 2 diabetes mellitus rises above $14 \mathrm{mmol} / \mathrm{l}$ requiring insulin correction. After 10 days of lowmolecular heparin therapy, the patient was switched to tableted anticoagulants, rivaroxabanum $15 \mathrm{mg}$ po. Dexamethasone was used during the whole period of respiratory failure.

On day 12 of the treatment, positive dynamics was observed: the body temperature was constantly normal $\left(36.5^{\circ} \mathrm{C}\right)$, weakness reduced, but still persisted, there was no cough and bilateral chest pain during it.

Laboratory findings demonstrated reduction of the cytokine storm (C-reactive protein, IL-6, procalcitonin and ferritin), normal white blood cells $9.0 * 109 / 1$, monocytosis $13.7 \%$ proving that inflammatory process comes to an end.

Pulse oximetry showed $98 \%$ oxygen saturation. $\mathrm{X}$-ray on 21st day of the disease (fig. 4) demonstrated positive development in complete decomposition of the obscure regions on the both sides with preservation of the diaphragm fibrosis.

Chest CT (fig. 5) findings on the 8th day after recovery showed single spots of pulmonary tissue on both sides according to the type of GGO, the total failure area of $5 \%$ in the sub-pleural sections of the lungs.

Despite the comorbid pathology, taking account absence of treatment at the pre-hospital stage, there was no major lung tissue damage. Thus, the inpatient treatment proceeded without any complications and negative dynamics.

After recovery, pulmonary rehabilitation was recommended. Physical activity should be dosed and only in the comfort zone without feeling fatigue and shortness of breath. Vaccination against pneumococcal infection once every five years and against influenza annually was recommended.

After all, numerous uncertainties remain in our understanding of the spread of COVID-19

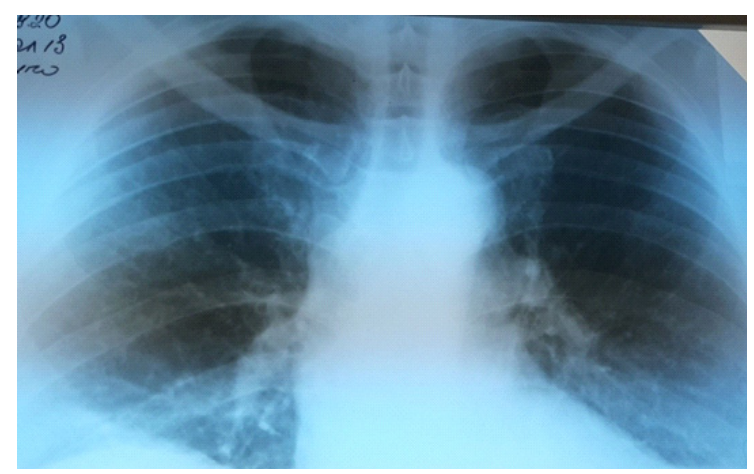

Fig. 4. X-ray

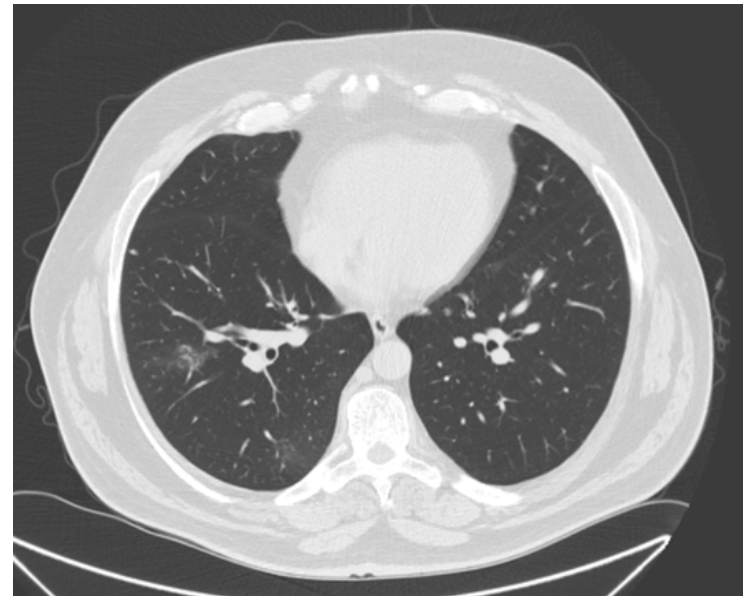

Fig. 5. Chest CT

and its management. There are limited data about reinfection with SARS-CoV-2 after recovery from COVID-19.

Acute lung injury and hyperinflammation in COVID-19 disease is associated with the presence of pulmonary fibrosis [12]. A pharmacological approach for reducing the risk of SARS-CoV-2 infection-associated pulmonary fibrosis or prevention of fibrotic status is investigated.

\section{Conflict of interests}

The authors of the article declare no conflict of interest.

\section{References}

1. Joseph S.M. Peiris, M.D. (December 18, 2003) The Severe Acute Respiratory Syndrome. N Engl J Med 2003 Vol. 349 No. 25; 2431-2441 DOI: 10.1056/NEJMra032498.

2. Rajesh T. Gandhi, M.D.( April 24, 2020) Mild or Moderate Covid-19. The New England Journal of Medicine. Vol. 383 No. 18. Retrieved from https://www.nejm.org/doi/full/10.1056/ NEJMcp2009249?query=featured_coronavirus

3. World Health Organisation. Coronavirus Disease (COVID-19): Frequently Asked Questions. Retrieved from https://www.who.int/ru/emergencies/diseases/novel-coronavirus-2019/advice-for-public/q-acoronaviruses.

4. Current information about coronavirus and quarantine. COVID-19 pandemic in Ukraine. Retrieved from https://covid19.gov.ua

5. Yen-ChinLiu, Rei-LinKuo, Shin-RuShih. (5 May 2020). COVID-19: The first documented coronavirus pandemic in history. Biomedical Journal. Volume 43, Issue 4, Pages 328-333. Retrieved from https:// www.sciencedirect.com/science/article/pii/S2319417020300445 
6. C Hani, N H Trieu, I Saab. (2020 Apr 3). COVID-19 pneumonia: A review of typical CT findings and differential diagnosis. 101(5):263-268. doi: 10.1016/j.diii.2020.03.014. Retrieved from https:// pubmed.ncbi.nlm.nih.gov/32291197/

7. Yujun Tang, Jiajia Liu,Dingyi Zhang. (2020 Jul 10). Cytokine Storm in COVID-19: The Current Evidence and Treatment Strategies. Front Immunol. Volume 11. doi: 10.3389/fimmu.2020.01708/ Retrieved from https://www.ncbi.nlm.nih.gov/pmc/articles/PMC7365923/

8. Dina Ragab, Haitham Salah Eldin. (16 June 2020). The COVID-19 Cytokine Storm; What We Know So Far. Front. Immunol. https://doi.org/10.3389/fimmu.2020.01446 Retrieved from https:// www.frontiersin.org/articles/10.3389/fimmu.2020.01446/full

9. Kimberly E. Hanson, Angela M. Caliendo, Cesar A. Arias (2020). Infectious Diseases Society of America Guidelines on the Diagnosis of COVID-19: Serologic Testing. Infectious Diseases Society of America. Retrieved from https://www.idsociety.org/practice-guideline/covid-19-guideline-serology/

10. Marina Carotti, Fausto Salaffi,Piercarlo Sarzi-Puttini. (2020 Jun 4). Chest CT features of coronavirus disease 2019 (COVID-19) pneumonia: key points for radiologists. Radiol Med. 1-11. doi: 10.1007/s11547020-01237-4 Retrieved from https://www.ncbi.nlm.nih.gov/pmc/articles/PMC7270744/

11. Guidelines of providing diagnostic and treatment in cases of COVID-19 of the Order of Ministry of Health of Ukraine No 762 dated 02/ 04 /2020 edited from 17 /09/ 2020 № 2116

12. Antonio Vitiello, Chiara Pelliccia, Francesco Ferrara. (2020Aug 27). COVID-19 Patients with Pulmonary Fibrotic Tissue: Clinical Pharmacological Rational of Antifibrotic Therapy. SN Compr Clin Med.: 1-4. doi: 10.1007/s42399-020-00487-7 Retrieved from https:/www.ncbi.nlm.nih.gov/pmc/articles/ PMC7452615/

Received: 11-Oct-2020

Accepted: 14-Dec-2020 